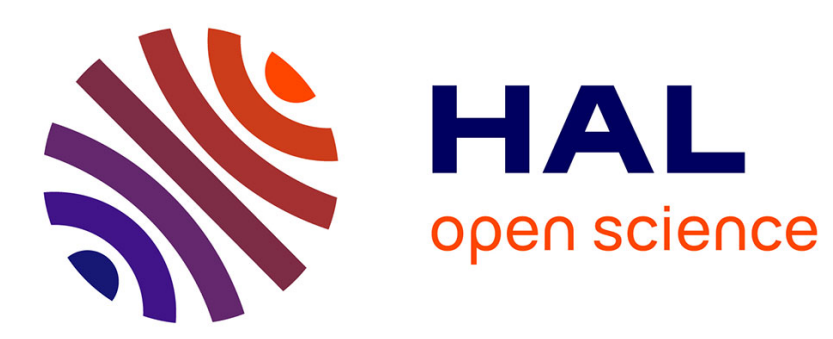

\title{
MIEUX COMPRENDRE LES PEURS FÉMININES : LA SOCIALISATION SEXUÉE DES ENFANTS AUX ESPACES PUBLICS URBAINS
}

Clément Rivière

\section{- To cite this version:}

Clément Rivière. MIEUX COMPRENDRE LES PEURS FÉMININES : LA SOCIALISATION SEXUÉE DES ENFANTS AUX ESPACES PUBLICS URBAINS. Sociétés contemporaines, 2019, 115, pp.181-205. 10.3917/soco.115.0181 . hal-03222283

\section{HAL Id: hal-03222283 \\ https://hal.science/hal-03222283}

Submitted on 10 May 2021

HAL is a multi-disciplinary open access archive for the deposit and dissemination of scientific research documents, whether they are published or not. The documents may come from teaching and research institutions in France or abroad, or from public or private research centers.
L'archive ouverte pluridisciplinaire $\mathbf{H A L}$, est destinée au dépôt et à la diffusion de documents scientifiques de niveau recherche, publiés ou non, émanant des établissements d'enseignement et de recherche français ou étrangers, des laboratoires publics ou privés. 


\title{
MIEUX COMPRENDRE LES PEURS FÉMININES : LA SOCIALISATION SEXUÉE DES ENFANTS AUX ESPACES PUBLICS URBAINS
}

\author{
Clément Rivière
}

Presses de Sciences Po | «Sociétés contemporaines»

2019/3 N 115 | pages 181 à 205

ISSN 1150-1944

ISBN 9782724636130

Article disponible en ligne à l'adresse :

https://www.cairn.info/revue-societes-contemporaines-2019-3-page-181.htm

Distribution électronique Cairn.info pour Presses de Sciences Po.

(C) Presses de Sciences Po. Tous droits réservés pour tous pays.

La reproduction ou représentation de cet article, notamment par photocopie, n'est autorisée que dans les limites des conditions générales d'utilisation du site ou, le cas échéant, des conditions générales de la licence souscrite par votre établissement. Toute autre reproduction ou représentation, en tout ou partie, sous quelque forme et de quelque manière que ce soit, est interdite sauf accord préalable et écrit de l'éditeur, en dehors des cas prévus par la législation en vigueur en France. Il est précisé que son stockage dans une base de données est également interdit. 


\title{
Mieux comprendre les peurs féminines : la socialisation sexuée des enfants aux espaces publics urbains
}

\author{
Clément Rivière
}

\begin{abstract}
Si l'accès plus difficile des femmes aux espaces publics urbains a été mis en lumière de longue date par la recherche en sciences sociales, la connaissance des processus socialisateurs qui sous-tendent cette différenciation sexuée demeure lacunaire. S'appuyant sur une enquête par entretiens menée auprès de 88 parents de 123 enfants âgés de 8 à 14 ans (69 garçons et 54 filles) à Paris (France) et Milan (Italie), cet article décrit la façon dont les parents encadrent de manière différenciée les usages que les enfants font de ces espaces selon le sexe biologique de ces derniers. Cette approche de l'action socialisatrice des parents apporte un éclairage empirique sur la sociogenèse des manières d'agir des femmes adultes dans les espaces publics urbains, l'encadrement parental contribuant à la formation de dispositions sexuées.

Mots-clés : Espace public - Genre - Socialisation - Enfants - Éducation
\end{abstract}

Better Understanding Women's Fears: The Gendered Socialization of Children to Urban Public Space

Unequal access of men and women to urban public space is old-established by social research. However, the understanding of the socialization processes underlying these gendered inequalities is incomplete. Drawing on in-depth interviews conducted in Paris (France) and Milan (Italy) with parents $(n=88)$ of children aged 8 to $14(n=123)$, this article focuses on parental supervision of children's urban practices. The analysis describes the transmission of different norms and representations according to children's biological sex and sheds an empirical light on the fabric of gendered uses of public space.

Keywords: Public space - Gender - Socialization - Children - Education

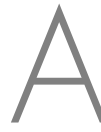

lors que les espaces publics urbains se définissent avant tout par leur libre accessibilité (Goffman, 2013 [1963] ; Joseph, 1984 ; Lofland, 1998), les conditions plus difficiles d'accès des femmes à ces espaces ont été mises en lumière de longue date par la recherche en sciences sociales ${ }^{2}$. De nombreuses recherches conduites en Amérique du Nord ont ainsi montré le caractère asymétrique de l'expérience masculine et féminine des espaces publics

1. Université de Lille, EA 3589 - CeRIES - Centre de recherche "Individus Epreuves Sociétés», clement.riviere@univ-lille.fr

2. L'auteur remercie Joanie Cayouette-Remblière, Virginie Dutoya, Camille Lancelevée, Gaspard Lion et les membres du comité de rédaction de Sociétés Contemporaines pour leur précieux regard critique sur de précédentes versions de ce texte. 
urbains, la vie publique urbaine des femmes se caractérisant notamment par l'expérience des male-to-female street remarks, ces remarques formulées par des hommes à destination exclusive des femmes qu'ils croisent dans la rue (Gardner, 1980). En décrivant un large éventail de pratiques de présentation de soi, d'évitement d'espaces, de recherche d'une escorte ou encore d'auto-enfermement, Carol Gardner a mis en lumière la charge mentale (general mental vigilance) et le travail de "préparation anticipatrice » associés à l'expérience urbaine des femmes (Gardner, 1990), en lien étroit avec la crainte d'être victime d'une agression sexuelle (Warr, 1985). En France, où le sentiment d'insécurité exprimé par les femmes est plus élevé que chez les hommes, notamment en milieu urbain (Lieber, 2011), l'Enquête Nationale sur les Violences Envers les Femmes en France (ENVEFF) a montré qu'une part significative des femmes (près de $40 \%$ ) évitent de sortir seules la nuit et qu'une part équivalente d'entre elles réfléchissent dans le détail à l'itinéraire qu'elles vont emprunter (Condon et al., 2005). Marylène Lieber a décrit ces pratiques de "contournement du risque ", qui peuvent par exemple consister à arriver en retard pour éviter de se trouver seule dans un lieu public, à emprunter un taxi ou sa voiture personnelle plutôt que les transports en commun, ou encore à s'habiller en évitant les tenues jugées «provocantes» ou moins propices à la fuite (Lieber, 2008). De manière générale, l'expérience urbaine des femmes se caractérise par un usage moins intensif des espaces publics que chez les hommes, en particulier la nuit. Alimentée par des « rappels à l'ordre » récurrents allant des compliments au harcèlement et à la violence, la "peur sexuée » associant féminité, espaces publics et danger (Lieber, 2008) invite à penser ces espaces comme des arènes d'exposition plus éprouvantes pour les femmes, dont les manières de se comporter, la présentation de soi, et en dernière analyse la simple présence, sont à tout moment susceptibles d'être remises en cause.

Les recherches portant sur les usages enfantins de la ville montrent que l'accès aux espaces publics urbains se différencie nettement dès l'enfance selon le sexe biologique, et cela dans des contextes urbains variés ${ }^{3}$. Pourtant, la connaissance des processus qui soustendent cette différenciation sexuée et les inégalités qui en découlent demeure lacunaire : les travaux portant sur l'expérience urbaine des femmes ont en effet souvent été réalisés par des géographes (Coutras, 1993 ; Di Méo, 2011) ou dans le cadre d'approches interactionnistes

3. Les garçons effectuent notamment plus souvent au même âge le trajet pour et depuis l'école de manière autonome, tandis que les filles se déplacent moins souvent seules et jouent moins longtemps sans surveillance dans les espaces publics aménagés à cet effet (voir par exemple Harden, 2000 ; Karsten, 2003a; Mc Millan et al., 2006). 
des comportements en public (Gardner, 1980 et 1990) peu enclines à l'interrogation des processus de socialisation, définis de manière large comme «l'ensemble des processus par lesquels l'individu est construit [...] par la société globale et locale dans laquelle il vit, processus au cours desquels l'individu [...] acquiert des façons de faire, de penser et d'être qui sont situées socialement» (Darmon, 2016, p. 6). De manière plus générale, l'étude des dimensions spatiales des processus de socialisation est un domaine de recherche en cours d'exploration (Authier, 2012) et peu de travaux empiriques se sont intéressés aux modalités concrètes de l'apprentissage des comportements en public en milieu urbain.

Poursuivant la réflexion engagée dans une publication précédente autour de la «fabrique des dispositions urbaines » au sein du cadre familial (Rivière, 2017), cet article s'appuie sur une enquête par entretiens conduite à Paris et Milan auprès de parents d'enfants âgés de 8 à 14 ans (voir encadré méthodologique) pour décrire les modalités et les principes de la différenciation sexuée de la socialisation à la ville des enfants. Alors que la transmission de compétences de mobilité et d'interaction, mais aussi de normes de comportement et de représentations du danger, s'effectue de manière distincte selon le volume et la composition des ressources dont disposent les parents (Rivière, 2017), il s'agit ici de s'intéresser à la façon dont la socialisation aux espaces publics urbains (définis comme les lieux publics et formellement accessibles à tous en milieu urbain ${ }^{4}$ ) se distingue selon le sexe biologique des enfants, en interrogeant l'existence de variations de classe à ce processus de différenciation sexuée.

Cette approche empirique du volet urbain du « travail parental » (Déchaux, 2009) et de l'action socialisatrice des parents permet de mieux «comprendre les peurs féminines» (Condon et al., 2005) en montrant le rôle que jouent les parents, et notamment les mères, dans la transmission d'un rapport sexué aux espaces publics urbains. En suggérant l'incorporation progressive de dispositions urbaines distinctes (ou pour le dire autrement de manières distinctes d'agir, de percevoir et d'évaluer dans les espaces publics urbains) selon le sexe biologique, elle contribue à sa mesure à une meilleure connaissance de la «fabrication sociale des individus » (Lahire, 2013).

4. Principalement le réseau viaire, les places, les parcs et les transports en commun (dont le caractère théoriquement payant de l'utilisation n'est pas nécessairement respecté). 


\section{Note méthodologique}

Cet article s'inscrit dans le prolongement d'une recherche portant sur les pratiques parentales visant à encadrer les déplacements et les activités des enfants dans les espaces publics urbains en contexte de mixité sociale (Rivière, 2014). Ce travail s'est principalement appuyé sur une campagne d'entretiens semi-directifs $(n=78)$ conduite entre mars 2009 et avril 2012 auprès de parents $(n=88)$ d'enfants âgés de 8 à 14 ans (54 filles et 69 garçons) à Milan (ltalie, 43 entretiens) et dans le $19^{\circ}$ arrondissement de Paris (France, 35 entretiens). D'une durée moyenne de 73 minutes, les entretiens ont été réalisés en français (à Paris) et en italien (à Milan), avant de faire l'objet d'une retranscription intégrale dans leur langue de réalisation, puis d'une analyse thématique. 61 mères et 27 pères ont été interrogés dans deux espaces péricentraux caractérisés par l'hétérogénéité de la composition sociale et culturelle de leur population ${ }^{5}$. Cette perspective de monographie comparée (Pinson, 2019), qui visait à mieux comprendre à partir de la mise en regard de deux enquêtes localisées la façon dont les contextes locaux contribuent à définir les pratiques d'encadrement parental, n'est pas développée dans l'article, dans la mesure où la socialisation sexuée des enfants aux espaces publics urbains s'est révélée transversale aux terrains d'enquête, de même que ses principes de variation de classe.

Le quadrilatère Villette-Belleville à Paris (125 393 habitants en 2008, dont $15,7 \%$ d'étrangers) et le triangle Monza-Padova à Milan (76 427 habitants fin 2008 , dont 23,7\% d'étrangers) partagent la même situation géographique dans les deux métropoles, à la périphérie nord-est de l'hypercentre. Ceci n'est pas sans lien avec le fait que leurs trajectoires de développement présentent de nombreux traits communs : ces anciens espaces agricoles et de villégiature, absorbés par la ville-centre dans le cadre de son expansion, ont successivement fait l'objet de processus d'industrialisation, de désindustrialisation puis de gentrification, moins avancée cependant sur le terrain milanais au moment de l'enquête (Rivière, 2014).

Les deux terrains se caractérisent donc à la fois par des formes d' "occupation populaire de la rue » (Clerval, 2011 ) et par une tendance à la hausse des prix immobiliers, en particulier à Paris. Celle-ci exerce une contrainte forte sur les familles, dans la mesure où la naissance puis la croissance des enfants engagent un rapport à l'espace domestique distinct de celui des célibataires ou des couples sans enfants (Vermeersch et al., 2019). La nette préférence de certains profils de parents pour un "style de vie urbain " (Karsten, 2003b) peut alors être remise en cause : en dehors du parc de logement social (résiduel dans le cas de l'espace étudié à Milan, et au sein duquel la rotation des locataires est faible à Paris), les familles qui résident à l'intérieur de la ville-centre sont déjà propriétaires de leur logement, disposent de moyens financiers conséquents ou arbitrent en défaveur de la taille de leur logement ou de l'accession à la propriété. Dans ce contexte, si le profil des parents rencontrés dans le cadre de l'enquête présente une certaine diversité, les parents des classes moyennes et moyennes-supérieures font l'objet d'une sur-représentation parmi les enquêtés, sans doute favorisée par la proximité

5. En 2008, année précédant le début de l'enquête, aucune des huit grandes catégories socio-professionnelles de l'INSEE (CSP) ne représentait ainsi plus d'un cinquième de la population active âgée de 15 ans et plus sur le terrain d'enquête parisien. Nettement sur-représentées par rapport au niveau national (20,5\% contre $8,7 \%$ ), les cadres et professions intellectuelles supérieures correspondaient au groupe le plus nombreux parmi les actifs, devant les employés (17,2\%) et les professions intermédiaires (16,7\%). À l'inverse, les ouvriers étaient sous-représentés par rapport au niveau national $(7,4 \%$ contre $13,1 \%)$, tandis que les artisans, commerçants et chefs d'entreprise pesaient à peu près le même poids démographique sur le terrain d'enquête et au niveau national $(2,8 \%$ contre $3,3 \%)$, de même que les employés $(17,2 \%$ et $16,4 \%)$ et dans une moindre mesure les professions intermédiaires (16,7 \% contre $13 \%)$. 
sociale avec l'enquêteur : un peu moins d'un tiers des enquêtés ont ainsi été classés a posteriori (sur la base de la profession déclarée ${ }^{6}$ comme faisant partie des "cadres et professions intellectuelles supérieures " (CS3 de l'INSEE), un peu plus d'un quart comme membres des professions intermédiaires (CS4), un cinquième comme employés (CS5), et un peu moins d'un dixième respectivement comme ouvriers (CS6) et artisans, commerçants et chefs d'entreprise (CS2).

Les parents ont été recrutés par le biais de quatre canaux principaux : démarchage dans des espaces publics (parcs, rues) ou semi-publics (centres d'animation à Paris, réunions publiques dans les deux villes, patronages paroissiaux à Milan), contact par l'intermédiaire d'informateurs-relais liés à des centres sociaux, des associations ou des lieux de culte, sollicitation par le biais d'associations de parents d'élèves, recommandation par un enquêté précédent. La recherche était dans les différents cas de figure présentée comme portant sur les usages enfantins des espaces publics dans des quartiers caractérisés par une population hétérogène.

La relative difficulté rencontrée à interroger des pères (27 enquêtés sur 88 ) est caractéristique des recherches portant sur les pratiques éducatives (voir notamment Barker, 2011 ; Lehman-Frisch et al., 2012), en lien avec l'implication plus importante des mères dans l'activité quotidienne de prise en charge des enfants. Cette sur-représentation des mères n'a pas été sans effets sur les relations tissées au cours de l'enquête, dans la mesure où l'enquêteur, alors âgé de 24 à 27 ans, sans enfant et de sexe masculin, était loin de " partager des contraintes de genre avec les enquêtées » (Le Renard, 2010). Si cette situation d'enquête n'a pas permis de jouer et de bénéficier d'une connivence avec les mères interrogées, la distance de genre ne constitue cependant "pas nécessairement une contrainte inéluctable ou fatale à l'enquête » (Gourarier, 201 1). Cette distance a en particulier souvent permis de faire preuve d'une ingénuité plus ou moins feinte (Bonnet, 2008) et de faire développer de manière détaillée certaines expériences des mères interrogées, à propos par exemple des "frotteurs » du métro ou des exhibitionnistes.

$\|$ convient enfin de préciser que les enfants et les adolescents sont susceptibles de mettre en œeuvre des pratiques de contournement des consignes et des injonctions parentales (Mardon, 2011 ; Bidet, 2018), raison pour laquelle il est périlleux d'inférer de manière mécanique les pratiques effectives des enfants des propos de leurs parents. Les pratiques d'encadrement parental n'en demeurent cependant pas moins l'un des principaux cadres (Lahire, 2013), si ce n'est le cadre central, de leur processus de socialisation aux espaces publics urbains.

\section{Représentations sexuées de l'aptitude à l'autonomie et de l'exposition des corps}

L'analyse des entretiens conduit à observer la forte prégnance de représentations sexuées de l'aptitude à la mobilité autonome des enfants, mais aussi de l'exposition de leurs corps dans les espaces publics urbains. De manière transversale aux propos des parents rencontrés sur les deux terrains, la rhétorique d'une plus grande « maturité » des filles au même âge est en particulier très prégnante (Valentine, 1997 ; Mardon, 2010).

L'analyse des entretiens conduit à observer la forte prégnance de représentations sexuées de l'aptitude à la mobilité autonome des enfants, mais aussi de l'exposition de leurs corps dans les espaces publics urbains. 
Que recouvre donc cette manière d'évoquer les différences perçues entre les enfants des deux sexes? Les propos des parents interrogés invitent à la construction d'un ensemble d'oppositions binaires en vue de mieux saisir ce que signifie « être mûr » à leurs yeux: les filles sont ainsi décrites comme davantage "attentives à leur environnement», par contraste avec des garçons « distraits ». Ces derniers ont « la tête en l'air » quand elles ont « les pieds sur terre »; elles sont plus « rationnelles » et « concrètes » alors qu'ils sont plus «imprévisibles », et leur « appréhension» de l'extérieur se distingue de l'«insouciance » des garçons. Quand les filles « racontent» et décrivent leur expérience quotidienne, les garçons « se taisent » et se réfugient dans un univers perçu comme enfantin.

Tout se passe comme si la « maturité » reconnue aux filles constituait une prime à leur prudence et à leur meilleur respect des règles (Granié, 2010) : l'ensemble des qualités qui leur sont associées les érigent en effet en utilisatrices des espaces publics intrinsèquement plus compétentes que les garçons, notamment pour faire face aux deux principales préoccupations parentales que sont les accidents de la circulation et la rencontre d'un inconnu malveillant (Rivière, 2018). Marcher « la tête dans les nuages» fait à l'inverse figure de repoussoir, comme de manière plus générale la moindre capacité perçue des garçons à se montrer attentifs à leur environnement. Les filles tendent ainsi à être considérées comme faisant preuve d'une meilleure aptitude à l'autonomie urbaine au même âge, en particulier en fin de scolarité primaire et à l'entrée dans le secondaire. Elles sont alors décrites comme davantage en mesure d'anticiper les situations potentiellement dangereuses, mais aussi d'y faire face, tandis que les garçons suscitent plus fréquemment de l'inquiétude chez leurs parents lorsqu'ils fréquentent les espaces publics sans eux. Une différenciation assez nette entre les pratiques urbaines des garçons et des filles se trouve ainsi opérée par les parents au cours des premières années de la tranche d'âge étudiée. Ce traitement différencié est parfois explicitement assumé, comme dans le cas d'Aliénor, une mère parisienne d'origine très favorisée qui n'autorise pas son fils à répliquer au même âge (dix ans) que sa sœur aînée l'organisation d'une fête d'anniversaire sans adulte dans un restaurant du quartier, impliquant des trajets nocturnes effectués de manière autonome par les convives:

- Aliénor: Pour ses dix ans, c'était la première soirée en fait, oui. C'était le cadeau d'anniversaire, elle avait demandé à la grand-mère de participer au dîner au resto, moi j'ai trouvé ça génial, en plus rien à préparer, pas de bazar à la maison, tout se passait dehors! [elle sourit] Ceci dit, quand le frère a demandé à faire ça, j'ai quand même dit : «Non, on va attendre », parce que les garçons sont moins... [ton évasif].

- CR : Tu fais moins confiance à tes garçons? 
- Aliénor: Oui, ils sont moins mûrs. Ils sont moins mûrs. Et eux-mêmes le savent. Alors après ça dépend peut-être des garçons, mais oui, c'est sûr. Ils vivent un peu dans un autre univers, les filles sont plus dans la réalité. Donc quand ils jouent ils sont dans un autre univers, ils ne voient pas ce qui se passe autour d'eux, ils ont moins l'idée de ce qui peut se passer, ils savent moins... Ils savent moins affronter... C'est ce que je te disais tout à l'heure, les filles vont repérer [elle insiste] si quelqu'un les suit, avoir des idées de ce qu'elles peuvent baratiner au suiveur, comment elles devraient réagir, en parler entre elles, les garçons non.

Aliénor, sans profession (mari chef d'entreprise), deux filles de 12 et 3 ans, deux fils de 11 et 8 ans (Paris).

La puberté et les transformations qui l'accompagnent sont régulièrement mobilisées pour expliquer la différence de maturité qui distinguerait garçons et filles. Comme l'a montré Aurélia Mardon, la puberté « change le regard» que les parents portent sur leurs filles, en particulier au moment des premières règles (Mardon, 2010). Cette évolution du regard parental dessine une sortie de l'enfance plus soudaine pour les filles, qui accèdent alors au statut de jeunes femmes, ce qui conforte la perception de leur «maturité ». Un tel jugement n'est d'ailleurs pas sans conséquence sur les autorisations accordées, nombre de parents leur faisant confiance plus tôt qu'aux garçons du même âge pour des pratiques de mobilité autonome diurnes.

Mais si les filles sont souvent considérées comme prêtes à se déplacer seules dans la ville à un âge où il conviendrait encore de protéger les garçons, cette confiance dans leur plus grande capacité à se déplacer de manière sûre cède progressivement la place à une peur associant avec une « connotation sexuée et sexuelle très claire » (Lieber, 2008) des dangers spécifiques au fait d'être une (jeune) femme dans les espaces publics urbains. Alors que la crainte d'un enlèvement, et en toile de fond celle de la rencontre d'un pédophile (Rivière, 2016), est tout autant prononcée dans le cas des garçons que dans celui des filles pour les enfants les plus jeunes de la tranche d'âge étudiée, l'analyse des entretiens permet d'observer la différenciation progressive des représentations de l'exposition des corps des filles et des garçons dans les espaces publics à mesure de leur avancée en âge, différents risques leur étant alors associés de manière spécifique.

Dans le cas des garçons, c'est l'exposition à la violence physique dans le cadre d'affrontements dépourvus de connotation sexuelle qui cristallise l'inquiétude, que cette violence soit provoquée ou subie par l'enfant. Les craintes des parents se renforcent notamment au moment du passage au collège, où l'exposition à la violence interpersonnelle est envisagée à la fois dans le contexte scolaire et dans les espaces publics, une certaine porosité entre ces deux arènes 
d'interaction n'étant pas exclue. Cette inquiétude spécifique aux garçons est liée à la perception d'une sociabilité se construisant davantage que celle des filles autour du rapport de force physique, tandis que l'éventualité que celles-ci puissent donner ou recevoir des coups n'est guère envisagée ${ }^{7}$. Cause ou conséquence de cette association aux garçons du recours et de l'exposition aux coups, leur initiation à l'usage de la force physique fait l'objet d'une attention nettement plus prononcée de la part des parents que dans le cas des filles (voir infra). Si le travail de terrain a permis à plusieurs reprises d'observer directement des interactions au cours desquelles les parents transmettent à leurs enfants des représentations sexuées de la force physique, conçue comme un attribut viril, son emploi n'a en revanche à aucun moment de l'enquête été envisagé comme pouvant être le fait de filles. Cette association exclusive au masculin de la force physique et de son emploi a pour effet d'entretenir chez les parents une crainte diffuse, relative à l'implication de leur(s) fils dans un affrontement physique subi ou provoqué. Leur force physique peut d'ailleurs être perçue comme si supérieure, qu'il s'agit de leur enseigner à ne pas y avoir recours contre les filles : envers du privilège et du devoir de protéger les filles, le déshonneur accompagne le recours à la violence exercée contre celles-ci.

Tout se passe dès lors comme si les filles étaient perçues comme structurellement mieux protégées de certaines formes de violence dans les espaces publics. Dans leur cas cependant, les préoccupations parentales se cristallisent autour de l'éventualité d'une agression sexuelle au moment de l'apparition des «attributs corporels de la féminité » (Mardon, 2010) : les transformations de leur corps à la puberté conduisent les parents à nourrir et exprimer de manière explicite des craintes qui leur sont spécifiques, relatives à leur vulnérabilité sexuelle présumée. L'accès au statut de jeune femme, intimement lié au changement du regard porté sur elles par leurs parents, a pour corollaire la découverte du traitement auquel les femmes sont confrontées dans le cadre de leur rapport ordinaire aux espaces publics. Dans le même temps, le risque d'une agression sexuelle visant les garçons devient progressivement un impensé. Audelà du fait que ceux-ci sont désormais supposés capables de se défendre face à l'attitude menaçante d'un adulte, l'éventualité qu'ils puissent susciter le désir d’agresseurs ne semble plus envisagée après la puberté, tandis que l'« ombre » de l'agression sexuelle (Ferraro, 1996) prend de la consistance dans l'esprit des parents de filles. Contribuant certes à les rendre plus « matures », les transformations

7. S'appuyant sur un échantillon représentatif de 2165 individus âgés de 17 ans et habitant Paris, l'enquête Escapad Paris 2010 (APUR, 2013) montre que plus d'un Parisien sur cinq âgé de 17 ans en 2010 avait déjà participé à une bagarre (22\%) contre moins d'une Parisienne sur 20 du même âge (4 \%). 
physiques dont ces dernières font l'expérience sont également perçues comme les rendant plus désirables et donc plus exposées, notamment dans les espaces publics.

\begin{abstract}
Ma fille aînée grandit un peu, donc les filles c'est un peu particulier aussi, parce que... Ben voilà, on fait un peu plus attention [...] Oui. Parce que les filles, ne serait-ce qu'à 14 ans, c'est des petites filles dans la tête et pas toujours... Physiquement, donc il faut quand même essayer de leur expliquer un petit peu... Pareil, les «dangers», entre guillemets, d'une vie urbaine. Alors qu'un garçon de 13-14 ans, on ne va peut-être pas avoir les mêmes...

Maud, expert-comptable (mari expert-comptable), deux filles de 13 et 10 ans, un fils de 7 ans (Paris).
\end{abstract}

Je pense que si j'avais un garçon je serais moins inquiète sur certaines choses. Notamment les agressions. Enfin, en même temps non, parce que les agressions, on sait que les garçons peuvent être agressés aussi, hein, j’ai des amis dont le fils, qui se balade avec un portable et un Ipod, s'est fait racketter plein de fois, il s'est fait agresser plein de fois. Récemment, là, par une bande, ils se sont jetés sur lui, ils l'ont racketté... Mais non, je pense que c'est différent. Du point de vue des agressions... physiques. Euh... [silence gêné] Te dire que je serais moins... Oui, je... Je pense que Lola peut être exposée à ce type d'agressions, en tant que fille, des agressions physiques... de la part de garçons, ouais, j'y pense, bien sûr. Si j'avais un garçon je penserais aussi à des agressions, mais d'un autre ordre.

Céline, chargée de projet dans une association (séparée), une fille de 11 ans (Paris).

La prégnance de ces représentations sexuées de l'exposition des corps dans les espaces publics confère des responsabilités différentes aux parents selon le sexe de l'enfant, dans la mesure où il s'agit de protéger de risques distincts, mais aussi car l'agression sexuelle est considérée comme nettement plus traumatisante. De manière transversale aux propos des parents rencontrés, la perception d'une plus grande vulnérabilité des garçons les plus jeunes cède ainsi la place, avec la puberté, à une peur associant des dangers spécifiques au fait d'être une (jeune) femme dans les espaces publics urbains.

\title{
Des arènes d'interaction perçues comme masculines
}

Les parents rencontrés à Paris et Milan anticipent pour la plupart d'entre eux des situations d'interaction asymétriques entre hommes et femmes dans les espaces publics urbains. L'insistance sur la probabilité forte de l'occurrence de tentatives de séduction des filles, centrales dans la performance de la masculinité hétérosexuelle (May, 2014; Gourarier, 2016), aide à comprendre pourquoi les « imprévus » (Goffman, 2002 [1977]) sont considérés comme nettement plus probables dans le cas des filles, mais aussi pourquoi les agresseurs imaginés ont pour point commun d'être toujours des hommes. 
Les expériences faites par les enfants des enquêtés permettent d'ailleurs à ces derniers de distinguer plusieurs types de situations d'interaction auxquelles les filles se trouvent plus fréquemment exposées. La rencontre d'individus se livrant à des pratiques exhibitionnistes a ainsi été mentionnée à plusieurs reprises au cours de l'enquête, mais jamais à propos d'un garçon. De manière plus générale, les filles sont davantage sollicitées dans le cadre d'approches relevant de tentatives de séduction. Nombre d'entre elles ont ainsi fait état à leurs parents de marques d'intérêt pour leur corps, de demandes relatives à leur prénom ou à leur numéro de téléphone, ou encore d'invitations diverses: elles semblent se faire bien plus souvent aborder, et parfois suivre, que les garçons. Les sollicitations auxquelles les filles se trouvent plus spécifiquement confrontées peuvent notamment s'effectuer sur le registre du « commentaire de rue » (Gardner, 1980) : quoi qu'il puisse être parfois perçu sur le mode du compliment, ce processus d'évaluation des corps et de la présentation de soi confronte les filles à des situations d'interaction auxquelles les garçons ne sont que rarement exposés, en tout cas en tant que récepteurs.

Dès le moment où je l'ai laissée se promener seule, elle a raconté des histoires de types louches, qui leur auraient, parce que souvent c'était avec des copines, c'était au début que [elle insiste] avec des copines, donc elle n'était pas seule, et elles avaient eu peur d'un homme qui les suivait. Alors, est-ce que c'est des fantasmes ou la réalité, j'ai tendance à croire que c'est plutôt la réalité. Et donc elles avaient à se... Ben, à gérer ça. Oui. Et, d'ailleurs, notre fille ça l'amusait presque. Enfin... C'était une lutte à mener de savoir triompher de ses angoisses et de ne pas avoir peur... Mais je sais qu'elle a une bonne copine qui n'est plus sortie toute seule pendant au moins un an, après avoir eu peur. D'un homme qui les aurait suivies.

Aliénor, sans profession (mari chef d'entreprise), deux filles de 12 et 3 ans, deux fils de 11 et 8 ans (Paris).

J'ai vu un jeune interpeller une fille, se mettre devant et presque l'embrasser. Une fille qu'il ne connaissait pas! Non... [ton de profond désaccord] En pleine journée ! [...] Oui, les filles sont plus facilement agressées, elles sont sifflées. Je vous dis, je l'ai vu, je l'ai vu de mes yeux, et puis après [il siffle], « Comment tu es? Jolie? Vas-y, t'es juive? T'es pas juive? ». Ça s'est déjà vu, ça, et elle avait pas 14 ans, elle avait 11 ans. [...] Après elles n'ont pas confiance.

Alexandre, chasseur de têtes pour un cabinet de recrutement (séparé), un fils de 9 ans et une fille de 7 ans (Paris).

Le recours à des entretiens conduits auprès de parents ne permet certes pas d'affirmer avec certitude qu'aucun de leurs fils n'ait jamais rencontré d'exhibitionniste, ou n'ait jamais été suivi, sifflé ou complimenté dans la rue ${ }^{8}$. On sait qu'il est plus difficile pour les garçons

8. D'après les premiers résultats de l'enquête Virage (Debauche et al. 2017), si les hommes sont près de quatre fois moins nombreux que les femmes à déclarer une agression sexuelle au cours de leur vie, plus de 
de faire part de sentiments de peur et de vulnérabilité (Goodey, 1997), et le fait que les filles tendent à davantage échanger à propos de leur vie quotidienne avec leurs parents (tout du moins sont-elles décrites ainsi par ces derniers) pourrait avoir comme conséquence de renforcer leurs craintes, en accentuant la perception des sollicitations dont elles peuvent faire l'objet. Quoi qu'il en soit, la perception selon laquelle les espaces publics sont davantage hostiles aux femmes, largement partagée par les enquêtés des deux sexes, s'appuie en grande partie sur l'expérience des mères. Structurés par la peur diffuse du viol, leurs récits et leurs pratiques confirment qu'elles associent les espaces publics à des dangers spécifiques pour les femmes, idée confortée par un nombre variable de situations auxquelles elles ont elles-mêmes été confrontées. Certains souvenirs de tentatives de séduction peuvent ainsi exercer une influence sur les autorisations accordées aux filles, comme en témoignent les propos d'Odile :

Pour revenir aux raisons pour lesquelles je ne veux pas qu'elles soient seules dans le parc, moi je ne suis pas très vieille, mais je ne suis pas très jeune non plus, j'ai un peu moins d'une quarantaine d'années, quand on est arrivé ici il m'est arrivé plusieurs fois, pour être très claire, de me faire draguer dans le parc. Il m'est arrivé d'y aller pour lire et de me faire draguer, même une fois en présence de mes enfants.

Odile, chargée de mission handicap en recherche d'emploi, deux filles de 14 et 11 ans, un fils de 12 ans (Paris).

Si les sifflements et autres commentaires de rue, auxquels les mères les plus jeunes sont particulièrement exposées, sont racontés de façon plus ou moins détachée ou inquiète, plusieurs enquêtées relatent avoir été suivies, ou fait l'objet de palpations vécues comme autant d'agressions. Sur les deux terrains d'enquête, les transports en commun, et en particulier le métro, sont associés de manière récurrente à ces « désagréments » (Lieber, 2008), à liimage des « gens qui se collent » évoqués par Morgane (aide-soignante, séparée, deux fils de 13 et 9 ans). L'expérience des mères ressort fortement des entretiens et s'érige en élément structurant de la perception du caractère masculin des espaces publics urbains, par contraste avec la relative insouciance dont les pères ont fait preuve au sujet de leur propre sécurité dans ces espaces. De fait, si nombre d'entre elles ont vécu comme Maria et Giovanna des expériences s'apparentant au harcèlement de rue au cours de leur adolescence, aucun père n'a jamais évoqué le fait d'avoir été suivi, d'avoir fait la rencontre d'un exhibitionniste, ou encore d'avoir fait l'objet d'attouchements ou de commentaires de rue : 
- Maria : C'était pareil pour moi à son âge, pour être honnête, des blondes aux yeux bleus... Quand j'avais 13 ans c'était quelque chose... C'était impossible d'aller faire un tour.

- CR : Vraiment ?

- Maria : J'étais une jolie fille quand j'étais plus jeune [ton ferme et assuré].

- CR : Je veux dire, on vous interpellait souvent?

- Maria : C'était... Vraiment, des choses vulgaires, que je n'ai parfois comprises qu'après, c'était impressionnant... [...] Et puis, bien entendu, celui qui te siffle, celui qui te suit dans la rue, ça m'est arrivé, oui, souvent, de très nombreuses fois même.

Maria, administratrice de biens immobiliers (mari chef d'entreprise), une fille de 12 ans, deux fils de 12 et 9 ans (Milan).

- Giovanna: Je me souviens que je descendais, je me souviens de ce jour-là parce que ça m'avait choquée, je descendais les marches de l'escalier du métro, et il y a un homme qui a ouvert, mais vraiment comme dans les films, qui a ouvert son imperméable et qui était nu en-dessous, j’avais 16 ans, et je suis restée pendant deux jours..

- CR: Choquée?

- Giovanna: Oui. Mamma mia..., et toutes les fois suivantes, mais pendant longtemps, hein, que j’ai emprunté cet escalier du métro, je me disais « Mamma mia, pourvu que je ne rencontre personne », parce que ça m'avait vraiment troublée. Et, bon, si ça pouvait arriver à l'époque, ça doit pouvoir arriver aujourd'hui.

Giovanna, institutrice (mari chauffeur de taxi), deux filles de 16 et 14 ans, un fils de 10 ans (Milan).

Il convient de prêter attention à la "socialisation des socialisateurs " pour comprendre pourquoi les filles sont perçues par les parents comme plus exposées aux regards et aux sollicitations indésirables que

les garçons, et comme devant en

conséquence

davantage

contrôler leur présentation de soi et leurs manières d'agir.

Il paraît dès lors nécessaire de prendre en compte les expériences que les mères elles-mêmes font et ont fait des espaces publics à différentes étapes de leur parcours de vie. En d'autres termes, il convient de prêter attention à la «socialisation des socialisateurs » (et notamment à la socialisation des socialisatrices) pour comprendre pourquoi les filles sont perçues par les parents comme plus exposées aux regards et aux sollicitations indésirables que les garçons, et comme devant en conséquence davantage contrôler leur présentation de soi et leurs manières d'agir. À l'inverse, les garçons ne sont plus considérés comme susceptibles d'être exposés à des avances d'ordre sexuel après la puberté: tout se passe comme si la nette prédominance des normes hétérosexuelles dans les espaces publics urbains (Blidon, 2008 ; Leroy, 2010) protégeait les garçons pubères en les préservant de l'occurrence de pratiques de séduction trop entreprenantes, coûteuses à mettre en œuvre aussi bien pour une (jeune) femme que pour un (jeune) homme. Cette perception partagée d'une exposition distincte des enfants pubères des deux sexes dans les espaces publics urbains sous-tend largement le traitement différencié dont filles et garçons font l'objet : cette différenciation s'opère de manière transversale aux familles rencontrées, même si des variations sociales à la socialisation sexuée doivent être mises en lumière. 


\section{Socialisation sexuée et variations de classe}

La prégnance de la perception des espaces publics urbains comme arènes d'interactions à dominante masculine a pour conséquence un encadrement plus strict des activités et des déplacements réalisés par les filles au sein de ces espaces après la puberté, de manière transversale aux familles rencontrées dans le cadre de l'enquête. Elles font alors l'objet d'un traitement différencié largement intentionnel, qui passe notamment par la transmission de normes de comportement et de présentation de soi spécifiques. L'enquête confirme la « dimension d'évidence » (Lieber, 2008) de la vulnérabilité des femmes en milieu urbain : une sérénité moindre que dans le cas des garçons se dégage des entretiens au sujet des filles pubères, qui se voient rappeler de manière fréquente les dangers spécifiques auxquels leur corps serait exposé dans les espaces publics urbains. Il s'agit alors pour les parents interrogés d'enseigner ou d'intimer à ces dernières de faire preuve de vigilance et de prudence, de ne pas «provoquer » en attirant l'attention, en somme de faire preuve de modération vestimentaire mais aussi dans le cours de l'interaction, notamment en sachant rester impassible face aux critiques et commentaires (Rivière, 2018).

Cette appréhension plus marquée des espaces ouverts à tous dans le cas des filles tend cependant à s'inscrire dans des registres de justification distincts en fonction des propriétés sociales des familles. La construction d'une opposition idéale-typique donne à voir «le nuancier des petites différences dans les socialisations de classe » (Darmon, 2016, p. 38) : plutôt mobilisé par les parents les moins dotés en capital culturel et scolaire, un registre naturalisant la différence de sexe s'oppose ainsi à un registre plus constructiviste et critique, caractéristique des parents du pôle «culturel » des classes moyennes et supérieures.

Dans le premier type, la plus grande dangerosité perçue des espaces publics urbains pour les filles (et de manière plus générale pour les femmes) ne fait guère l'objet de discussions, et la différenciation sexuée des usages de la ville n'est pas questionnée. Les différences observées peuvent même dans certains cas être associées à des causes biologiques, comme dans le cas de Vincente (encadrantcourrier à La Poste, deux fils de 13 et 11 ans, une fille de 8 ans, Paris), qui les interprète à l'aune de discours « scientifiques » relatifs à l'existence de cerveaux masculins et féminins. La force physique est alors toujours associée au masculin, comme le montre l'interaction qui suit entre Jamila et son fils aîné. Non-diplômée et allocataire du RSA, cette jeune veuve originaire d'Algérie et mère de deux 
garçons (âgés de 8 et 6 ans) réplique fermement à son aîné, venu interrompre l'entretien pour lui montrer un «bobo» : «Ce n'est pas grave, tu es un homme, tu es fort! », avant de se tourner vers moi comme pour chercher confirmation : «Ben oui, les hommes ils sont forts normalement!» (entretien du 17 juin 2009, Paris). Comme l'a montré Isabelle Clair au sujet du rôle joué par les " grands frères » dans les quartiers d'habitat social (Clair, 2008), la protection physique des filles est alors souvent confiée aux garçons, l'inverse ne se vérifiant qu'au sujet de garçons en bas âge ayant une sœur aînée. De manière révélatrice, Sophia (titulaire d'un BEP en comptabilité) attend ainsi de son fils qu'il « défende» sa sœur cadette d'un an, pourtant sensiblement plus grande et plus lourde que lui :

Ben là le fait qu'on déménage, et que, ben ma fille va se retrouver en $6^{e}$ et mon fils en $5^{e}$, ils seront dans le même collège, donc il faudrait que mon fils prenne un peu plus d'assurance parce que... Je ne sais pas si c'est le fait qu'il soit petit physiquement qui le frustre un petit peu, c'est possible. Mais ça reste le grand frère, c'est ce que je lui dis à chaque fois [...] [nouveau passage des enfants, qui ont fini leur goûter] Vous voulez aller en bas [NB : du parc où l'entretien est en cours de réalisation] ? Tu fais attention à ta sœur?

Sophia, éboueure à la ville de Paris (séparée), fils de 12 ans et une fille de 11 ans.

Ces pratiques sexuées sont caractéristiques du style d'encadrement parental « protecteur » (Rivière, 2017), qui a pour objectif principal de protéger les enfants des menaces extérieures, et se caractérise à la fois par une anticipation faible de la prise d'autonomie de l'enfant et par un intérêt limité pour la transmission de compétences de mobilité. Dans le second type à l'inverse, un travail de sensibilisation spécifique aux filles est mis en œuvre par les parents sur le registre de la déploration, mais justifié par souci de "réalisme» en dépit d'une sensibilité plus grande aux questions de genre et d'une conception égalitariste plus ou moins revendiquée des rapports sociaux de sexe. Ce travail de préparation à l'occurrence probable de situations susceptibles de surprendre ou d'embarrasser les filles, comme le fait d'être regardée, complimentée, sifflée, voire touchée ou suivie, s'inscrit dans la logique « préparatrice » caractéristique des styles d'encadrement parental des classes moyennes et supérieures (Rivière, 2017). Sur le terrain milanais, où les hommes immigrés, associés à des manières spécifiques de se comporter dans les espaces publics, notamment vis-à-vis des femmes, cristallisent les craintes pour les filles, les enquêtés les mieux dotés en capital culturel et scolaire tendent ainsi à moins s'alarmer, et disent essayer de "préparer » leurs filles à ces types spécifiques d'interaction. C'est notamment le cas de Tommaso, journaliste free-lance, qui évoque un «travail » à réaliser auprès de ses deux filles, respectivement âgées de huit et cinq ans au moment de l'entretien : 
Mes filles sont très blondes et les Maghrébins, même si elles sont encore petites, ont tendance à faire des commentaires, qui peuvent être positifs d'ailleurs. Quand ils voient une petite blonde, ils ont du mal à ne pas te dire qu'elle est jolie, etc. On voit vraiment que c'est quelque chose qui les étonne, qui leur plait, et il n'y a rien de mal, dans le sens où... Ce qu'il faut c'est leur dire, leur faire comprendre [à ses filles]. C'est un travail qu'il va falloir qu'on fasse [...] Le commentaire de rue sexiste est une réalité. Et donc une fille de 16 ans, ou de 14 ans, blonde, qui se balade dans la rue, n'est pas menacée, car je ne le vis pas comme une menace, mais est sans aucun doute exposée à davantage de sollicitations extérieures qu'un garçon du même âge. C'est comme ça. Et donc pour elles c'est un peu plus compliqué.

Tommaso, journaliste free-lance (femme graphiste), deux filles de 8 et 5 ans (Milan).

Une autre facette du «travail parental » (Déchaux, 2009) spécifique aux filles est mise en œuvre de façon contrastée selon les profils des familles. Il s'agit du contrôle de l'habillement, évoqué de manière exceptionnelle au sujet des garçons ${ }^{9}$, mais qui constitue une dimension saillante de l'expérience des parents de filles, comme l'illustre le cas d'Odile, qui décrit la façon dont elle «travaille sur l'habillement » de ses deux filles (mais pas de son fils):

Bon, par exemple, je travaille sur l'habillement, des choses comme ça : «Tu ne te promènes pas en jupe les jours où personne ne t'accompagne ». Elles se mettent en robe et en jupe le week-end [elle rit]. C'est élémentaire, mais c'est... Voilà. C'est pareil, mettre un décolleté, ok, mais quelle profondeur? Et est-ce qu'on met une écharpe ou pas? Non mais... C'est un peu... C'est concret, mais justement, enfin, il faut parler concret et dire clairement les choses.

Odile, chargée de mission handicap en recherche d'emploi, deux filles de 14 et 11 ans, un fils de 12 ans (Paris).

Comme l'a observé Aurélia Mardon au sujet de collégiennes des Hauts-de-Seine (Mardon, 2011), le contrôle exercé sur l'habillement des filles semble plus systématique dans les familles des classes moyennes et supérieures, un effort de transmission des « normes de la bonne mesure vestimentaire » (Mardon, 2010) s'opérant en vue de limiter le risque que les filles ne se fassent remarquer, importuner et éventuellement agresser. Si ces parents tendent de manière générale à promouvoir des normes de présentation de soi dans les espaces publics urbains qui s'articulent autour d'un idéal de discrétion (Rivière, 2017), les injonctions à la discrétion sont davantage prononcées encore dans le cas des filles, les styles vestimentaires jugés trop «féminins » (jupes jugées trop courtes, décolletés jugés trop échancrés...) étant érigés en facteur de danger. S'inscrivant clairement à l'encontre de ces recommandations, la tendance contemporaine à l'érotisation des corps des jeunes filles (Liotard et

9. Au sujet du port de pantalons «taille basse », qui dévoilent en partie leurs sous-vêtements (voir également Court et Mennesson, 2015). 
Jamain-Samson, 2011) paraît peu compatible avec les préoccupations de ce profil de parents, ce qui ne va pas sans créer des tensions entre les parents et les filles (Mardon, 2011), mais aussi dans l'esprit des parents eux-mêmes, en particulier dans le cas des mères qui font état de contradictions entre leur idéal d'égalité et leurs pratiques concrètes d'encadrement. L'entretien réalisé avec Bianca est à ce titre éclairant : alors que cette chercheuse dans une prestigieuse université publique milanaise, titulaire d'un doctorat en urbanisme, se présente comme s'étant «toujours» donné pour objectif de prodiguer une éducation non genrée à ses enfants, elle se sent contrainte par les goûts et les demandes vestimentaires de sa fille à exercer un travail de contrôle de l'habillement qu'elle ne met jamais en cuuvre dans le cas de son fils :

J'ai toujours cherché à élever mes enfants exactement de la même manière, naturellement. Même si je me suis rapidement rendue compte qu'une application un peu idéologique de ce projet se heurte au fait que non seulement chaque enfant est unique au sein de son propre genre, mais aussi qu'il y a de grandes différences entre garçons et filles. Sur certaines choses, par exemple, les tâches ménagères, il ne doit y avoir aucune différence, c'est quelque chose qu'on ne peut même pas discuter. Sur tout un ensemble de sujets, ils sont élevés exactement de la même manière tous les deux. Mais sur d'autres sujets, c'est plus compliqué, parce que ce sont eux-mêmes... Par exemple, mon fils n'accorde aucune importance à la façon dont il est habillé. Vraiment aucune. Ma fille, en revanche, cela fait un moment que son habillement fait l'objet de discussions quotidiennes... Elle veut imiter ses amies et les mannequins qu'elle voit à la télévision et moi je trouve que, même si elle est encore petite, ça devient facilement un peu provoquant, je ne sais pas comment le dire, mais ça t'oblige à faire un peu attention, même si tu as envie de dire : "Pour moi vous êtes égaux, je ne fais aucune différence ». Tu fais un peu plus attention parce que, par exemple, ma fille, nous n'avons pas la télévision, donc elle n'a pas directement l'occasion de la regarder mais ces modèles arrivent très vite dans toutes les maisons, parce que ses amies regardent les chanteuses, etc., et donc quelquefois j'ai l'impression que c'est un habillement un peu trop... Un peu limite. Tant qu'elle a 10 ans personne ne s'en rend compte, mais quand elle en aura quatorze... J'y penserai à deux fois avant de la laisser sortir habillée comme ça.

Bianca, chercheuse en urbanisme (mari chef d'entreprise), une fille de 10 ans et un fils de 9 ans (Milan).

Il ne faudrait cependant pas déduire des développements qui précèdent que la supervision de l'habillement des filles est caractéristique d'un profil de parents. Plusieurs mères appartenant aux catégories intermédiaires et populaires ont ainsi insisté au cours des entretiens sur leur volonté que leur fille ne "fasse pas Lolita», en exprimant l'idée qu'une présentation de soi sexualisée constitue une source de danger potentiel dans les espaces publics urbains. Si c'est bel et bien dans les familles les plus favorisées qu'il semble le plus systématique, le travail de veille vestimentaire existe dans la plupart des familles rencontrées, même s'il est parfois délégué à d'autres 
membres de la famille que les parents (grands-parents, frères et sœurs aînés) :

J'aimerais pas qu'elle soit habillée comme une lolita, ça c'est sûr. Je pense que ses frères feraient très attention à ce que... De toute façons, quand on a des frères aînés, vous savez, la vie est très dure pour les filles [rire franc], donc je pense que ça va être assez surveillé par ses frères.

Vincente, encadrant-courrier à La Poste (séparée), deux fils de 13 et 11 ans, une fille de 8 ans (Paris).

Si des variations de classe se dessinent, les pratiques différencialistes semblent ainsi revêtir un caractère transversal à l'espace social. Alors que des enquêtes récentes sur le sentiment d'insécurité suggèrent que l'on a plus souvent peur pour ses enfants que pour soi, en particulier en ce qui concerne la fréquentation de la rue et des transports en commun (Robert et Zauberman, 2017), on peut faire l'hypothèse que la socialisation aux espaces publics urbains est un domaine dans lequel l'indifférenciation souvent prônée par les parents est plus difficile à mettre en œuvre que dans d'autres domaines. Les filles demeurent en effet associées à des risques spécifiques et se voient régulièrement rappeler leur vulnérabilité supposée dans ces espaces, et cela quel que soit leur milieu social. Titulaire d'un DEA en psychologie, Odile (Paris, deux filles de 14 et 11 ans, un fils de 12 ans) déclare ainsi sans ambages: "Moi je suis plus inquiète pour mes filles ». Maîtresse de conférences à l'université et titulaire d'un doctorat d'allemand, Catherine (Paris, deux fils de 13 ans et 1 an, une fille de 9 ans) pense que son conjoint (maître de conférences lui aussi) et elle « auront plus d'appréhension pour Irène, certainement [que pour leur fils] ». Consultant en informatique et diplômé d'une école d'ingénieur, François (Paris, une fille de 12 ans, deux fils de 9 ans) affirme que « les raisons de sécurité priment » pour justifier le fait que les horaires de retour de sa fille seront probablement « très encadrés», plus en tout cas que dans le cas de ses fils, bien qu'il juge ces derniers «moins matures» au même âge.

Le fait que ces jugements s'opèrent sur un registre plus ou moins critique ou naturalisant n'empêche en rien la mise en œuvre de pratiques d'encadrement spécifiques aux filles. Dans l'ensemble des familles rencontrées, la tombée de la nuit constitue en particulier un obstacle à leur mobilité autonome, différentes sortes de restrictions étant mises en œuvre de manière plus drastique que dans le cas des garçons, à l'image d'interdictions de sortir, d'horaires de retour à respecter ou encore de pratiques d'escorte par les parents, par d'autres adultes ou par des pairs adolescents. Ne pas laisser se déplacer une fille seule le soir dans les espaces publics urbains correspond à une norme partagée par la plupart des enquêtés, mais aussi par leur entourage amical et familial. Marta (institutrice, un fils 
de 11 ans et une fille de 5 ans, Milan) regrette ce qu'elle décrit comme un état de fait : "Pour moi un garçon peut objectivement rentrer seul chez lui le soir vers 14-15 ans. Une fille non ». L'emprunt autonome des transports en commun le soir suscite en particulier moins d'appréhension dans le cas des garçons : le convoyage des trajets par l'un des parents est bien plus fréquent dans le cas des filles, que celui-ci soit intégral ou partiel (certains parents vont ainsi à la rencontre de leurs filles dans le métro afin qu'elles ne terminent pas seules un trajet débuté en compagnie d'amies ou d'amis). Cette volonté d'encadrer de manière plus stricte le retour des filles au domicile rend leurs sorties nocturnes plus difficiles à négocier, dans la mesure où ces sorties exigent alors la disponibilité d'un parent pour les escorter.

- Hélène : Donc on se donnait des fois rendez-vous à la gare du Nord, pour finir le trajet en métro, des choses comme ça.

- CR : Vous alliez l'attendre au métro ? [surpris].

Hélène : Oui, ça s'est trouvé. Parce qu'elle remontait en général en RER, donc je lui disais: "Si tu veux, je viens à ta rencontre».

$[\ldots]$

- CR : Donc la nuit ça vous inquiète toujours un peu?

- Hélène : Oui quand même, oui. Ben parce que c'est des filles aussi.

Hélène, assistante sociale (mari médecin salarié, deux filles de 19 et 16 ans, un fils de 16 ans (Paris).

La prégnance de pratiques d'encadrement qui visent à contrôler les pratiques de mobilité et de présentation de soi des filles contraste avec l'absence de discours s'inscrivant dans une perspective d'empowerment.

La prégnance de pratiques d'encadrement qui visent à contrôler les pratiques de mobilité et de présentation de soi des filles contraste avec l'absence de discours s'inscrivant dans une perspective d'empowerment. Aucun des parents de filles rencontrés (46 parents concernés pour la phase d'âge 8-14 ans, dont 32 mères) n'a ainsi fait la démarche de leur apprendre à «se défendre " (Dorlin, 2017). Pour le dire autrement, le travail de préparation à l'interaction mis en œuvre par les parents de filles les mieux dotés en capital culturel et scolaire ne va pas jusqu'à l'apprentissage de l'autodéfense. Ce constat est d'autant plus étonnant que l'on observe que les parents sont moins inquiets pour les filles capables de courir vite ou d'avoir recours à la force physique, du fait de leur morphologie ou de certaines de leurs expériences extra-scolaires: la "peur sexuée » (Lieber, 2008) s'atténue pour les filles dont les pratiques et les ressources propres suggèrent une moindre vulnérabilité. Celles-ci sont alors considérées et traitées d'une manière proche de celle des garçons.

- CR : Ce sont toujours des garçons qui raccompagnent les filles chez elles le soir?

- Giovanna : Oui, oui. Mais il y a une fille du quartier qui est au lycée qui a l'autorisation de se balader seule, elle doit faire quelque chose comme deux mètres et peser 80 kilos, elle fait de l'aviron, c'est une géante qui fait un peu 
peur, alors quand elle dit: «Je l'accompagne!» [NB : leur fille cadette], ça nous va aussi [elle rit].

Giovanna, institutrice (mari chauffeur de taxi), deux filles de 16 et 14 ans, un fils de 10 ans (Milan).

Peut-être les filles dont il est ici question sont-elles encore trop jeunes aux yeux de leurs parents pour apprendre à se défendre ou à remettre en cause les normes de genre ? Peut-être ces pratiques se diffusent-elles progressivement au cours des années qui suivent, sans doute alors de manière distincte selon les profils des familles? Notons, quoi qu'il en soit, qu'aucun des parents rencontrés ne semble enseigner aux garçons comment se comporter de manière non sexiste dans les espaces publics urbains ${ }^{10}$.

En décrivant la façon dont les parents, considérés en tant qu'agents centraux de la socialisation aux espaces publics urbains des enfants, accompagnent et encadrent de manière différenciée la découverte et les usages que les enfants font de ces espaces selon leur sexe biologique, cet article constitue une nouvelle confirmation de l'existence de différents "genres de la socialisation spatiale » (Löw, 2015 [2001]).

Alors que la naturalisation des différences produites par les processus de socialisation sexuée s'explique largement par le fait que ceux-ci débutent dès le plus jeune âge (Martin, 1998) voire avant même la naissance (Pélage et al., 2016), l'analyse des entretiens conduits auprès des parents rencontrés à Paris et Milan aide à ne pas «placer du côté de la nature ce qui peut être pensé comme le produit d'une intériorisation par petites touches insensibles, répétées, parfois volontaires, et parfois involontaires» (Lahire, 2007 [2005]). Elle apporte un éclairage empirique sur la sociogenèse des manières d'agir et de percevoir des femmes adultes dans les espaces publics urbains, et notamment sur les précautions qu'elles prennent, qui recoupent largement celles mises en œuvre par les parents pour leurs filles (évitement de lieux et de moments, recherche d'une escorte, gestion de la présentation de soi).

Cette mise en lumière de l'existence de différentes « classes de socialisation» (Darmon, 2016) aux espaces publics urbains, liée à la transmission de "manières de voir, de dire, de sentir et d'agir » sexuées (Lahire, 2001), invite à essayer d'articuler les processus de socialisation sexuée et leurs variations de classe. Alors que les parents les moins dotés en capital culturel et scolaire aspirent avant tout à «protéger» leurs filles sur le registre de la naturalisation de la 
En distinguant le champ des possibles et les conditions dans lesquelles les filles et les garçons peuvent explorer leur espace de résidence et à plus

large échelle la ville,

l'encadrement parental contribue selon toute vraisemblance à la formation de dispositions sexuées. différenciation des expériences, ceux qui en sont les mieux dotés tendent plutôt à mettre en avant leur volonté d'anticiper l'asymétrie des rapports de genre dans les espaces publics pour «préparer » leurs filles à des risques présentés comme objectifs. S'ils questionnent la division sexuelle des rôles sociaux, ils ne mettent pas moins en œuvre des pratiques d'encadrement spécifiques pour leurs filles, qui s'articulent autour de cette asymétrie qu'ils peuvent par ailleurs déplorer. De manière révélatrice de l'écart qui subsiste plus généralement entre les discours des parents critiques à l'égard des stéréotypes de sexe et leurs pratiques réelles (Dafflon-Novelle, 2006; Pélage et al., 2016 ; Court, 2017), la substance du message transmis aux filles se distingue en fin de compte assez peu: de façon transversale aux familles rencontrées, celles-ci sont socialisées à l'évitement des interactions et à la discrétion, mais pas à la remise en cause des normes de genre.

En distinguant le champ des possibles et les conditions dans lesquelles les filles et les garçons peuvent explorer leur espace de résidence et à plus large échelle la ville, l'encadrement parental contribue selon toute vraisemblance à la formation de dispositions sexuées, ces «manières durables d'agir, de penser et de percevoir, socialement codées comme féminines ou masculines et attendues d'un sexe plus que de l'autre » (Court, 2017), et à l'acquisition d'un rapport plus ou moins assuré et confiant aux espaces publics urbains selon le sexe biologique. S'il convient de garder à l'esprit qu'il s'agit d'une « grande illusion » de penser que « le savoir demeure inchangé dans le processus de transmission de l'émetteur vers le récepteur » (Lahire, 2007 [2005]), des recherches directement conduites auprès d'enfants confirment que les préoccupations que nourrissent les garçons et les filles au sujet de leur sécurité dans les espaces publics sont distinctes, ces dernières ayant notamment fortement intériorisé le risque spécifique de l'agression sexuelle (Harden, 2000).

Théâtres de la production continue du masculin et du féminin, les espaces publics urbains doivent dans cette perspective être considérés comme l'un des différents « espaces de construction du genre » dont Isabelle Clair, qui évoque le travail et la sexualité, fait l'hypothèse de l'existence (Clair, 2012). Et cela bien au-delà du cas le plus étudié, dans le contexte français, des quartiers périphériques d'habitat social (voir notamment Clair, 2008 ; Lapeyronnie, 2008). 


\section{RÉFÉRENCES BIBLIOGRAPHIQUES}

APUR, 2013

Authier J.-Y., 2012

Barker J., 2011

Bidet J., 2018

Bonnet F., 2008

Blidon M., 2008

Clair I., 2008

Clair I., 2012

Clerval A., 2011

Condon S., Lieber M., Maillochon F., 2005

Court M., 2017

Court M., Mennesson C., 2015

Cousin B., 2008
Avoir 17 ans à Paris. Une exploitation des résultats de l'enquête Escapad Paris 2010, Paris : Atelier Parisien d'Urbanisme.

Espace et socialisation. Regards sociologiques sur les dimensions spatiales de la vie sociale, Sarrebrücken : Éditions universitaires européennes.

«"Manic Mums" and "Distant Dads" ? Gendered Geographies of Care and the Journey to School », Health and Place, 17, p. 413-421.

« Les frontières du genre. Descendantes d'immigrés algériens face aux décalages de normes en Algérie », Sociétés contemporaines, 110, p. 7-34.

« La distance sociale dans le travail de terrain : compétence stratégique et compétence culturelle dans l'interaction d'enquête », Genèses, 73, p. 57-74.

« La casuistique du baiser. L'espace public, un espace hétéronormatif », EchoGéo [en ligne], 5.

Les Jeunes et l'amour dans les cités, Paris : Armand Colin.

Sociologie du genre, Paris : La Découverte.

«L'occupation populaire de la rue : un frein à la gentrification ? L'exemple de Paris intra-muros », Espaces et sociétés, 144-145, p. 55-71.

«Insécurité dans les espaces publics: comprendre les peurs féminines », Revue française de sociologie, 46 (2), p. 265-294.

Sociologie des enfants, Paris : La Découverte.

« Les vêtements des garçons. Goûts et dégoûts parentaux au sein des classes moyennes », Terrains et travaux, 27, p. 41-58.

Cadres d'entreprise et quartiers de refondation à Paris et à Milan. Contribution à l'analyse différenciée du rapport des classes supérieures à la mixité socio-spatiale et aux dynamiques d'auto-ségrégation, thèse de doctorat en sociologie, Institut d'Etudes Politiques de Paris et Università degli studi di Milano-Bicocca. 
Coutras J., 1993

Dafflon-Novelle A. (dir.), 2006

Darmon M., 2016 [3éd.]

Debauche A., Lebugle A., Brown E., Lejbowicz T., Mazuy M., Charruault A., Dupuis J., Cromer S., Hamel C., 2017

Dechaux J.-H., 2009

Di Meo G., 2011

Dorlin E., 2017

Ferraro K., 1996

Gardner C. B., 1980

Gardner C. B., 1990

Goffman E., 2002 [1977]

Goffman E., 2013 [1963]

Goodey J., 1997

Gourarier M., 2011

Gourarier M., 2016

Granie M.-A., 2010
« La mobilité des femmes au quotidien : un enjeu des rapports sociaux de sexe? », Les Annales de la recherche urbaine, 60, p. 164-170.

Filles-garçons. Socialisation différenciée? Grenoble : Presses universitaires de Grenoble.

La Socialisation, Paris : Armand Colin.

« Présentation de l'enquête Virage et premiers résultats sur les violences sexuelles », INED, Documents de travail, 229.

« Travail parental et parenté : parlons-nous de la même chose ? », Informations sociales, 154 , p. 14-20.

Les Murs invisibles. Femmes, genre et géographie sociale, Paris : Armand Colin.

Se défendre. Une philosophie de la violence, Paris : La Découverte.

« Women's Fear of Victimization : Shadow of Sexual Assault ? », Social Forces, 75 (2), p. 667-690.

« Passing by : Street Remarks, Address Rights, and the Urban Female », Sociological Inquiry, 50 (3-4), p. 328-356.

«Safe Conduct: Women, Crime, and Self in Public Places », Social Problems, 37 (3), p. 311-328.

L'Arrangement des sexes, Paris: La Dispute.

Comment se conduire dans les lieux publics. Notes sur l'organisation sociale des rassemblements, Paris: Economica.

«Boys Don't Cry. Masculinities, Fear of Crime, and Fearlessness », British Journal of Criminology, 37 (3), p. 401-418.

« Négocier le genre? Une ethnologue dans une société d'hommes apprentis séducteurs », Journal des anthropologues, 124-125, p. 159-178.

«Séduction», dans Rennes J. (dir.), Encyclopédie critique du genre, Paris : La Découverte, p. $596-604$.

«Socialisation au risque et construction sociale des comportements de l'enfant piéton : éléments de réflexion pour l'éducation routière », Enfances, familles, générations, 12, p. 88-110. 
Harden J., 2000

Joseph I., 1984

Karsten L., 2003a

Karsten L., 2003b

Lahire B., 2001

Lahire B., 2007 [2005]

Lahire B., 2013

Lapeyronnie D., 2008

Lehman-Frisch S., Authier J.-Y., Dufaux F. (dir.), 2012

Le Renard A., 2010

Leroy S., 2010

Lieber M., 2008

Lieber M., 2011

Liotard P., JamainSamson S., 2011
«There's no Place Like Home: The Public/Private Distinction in Children's Theorizing of Risk and Safety », Childhood, 7 (1), p. 43-59.

Le Passant considérable. Essai sur la dispersion dans l'espace public, Paris : Librairie des méridiens.

« Children's Uses of Public Space. The Gendered World of the Playground », Childhood, 10 (4), p. 457-473.

« Family Gentrifiers : Challenging the City as a Place Simultaneously to Build a Career And To Raise Children », Urban Studies, 40 (12), p. 2573-2584.

« Héritages sexués : incorporation des habitudes et des croyances », dans Blöss T. (dir.), La dialectique des rapports hommes-femmes, Paris : PUF, p. 9-25.

« Prédispositions naturelles ou dispositions sociales? Quelques raisons de résister à la naturalisation de l'esprit », dans Lahire B., L'esprit sociologique, Paris : La Découverte, p. 261-307.

Dans les plis singuliers du social. Individus, institutions, socialisations, Paris : La Découverte.

Ghetto urbain. Ségrégation, violence, pauvreté en France aujourd'hui, Paris : Robert Laffont.

« Les enfants et la mixité sociale dans les quartiers gentrifiés à Paris, Londres et San Francisco », Caisse Nationale d'Allocations Familiales, Dossier d'études, 153.

« Partager des contraintes de genre avec les enquêtées. Quelques réflexions à partir du cas saoudien », Genèses, 81, p. 128-141.

« "Bats-toi ma sœur". Appropriation de l'espace public urbain et contestation de la norme par les homosexuels », Métropoles [en ligne], 8.

Genre, violences et espaces publics. La vulnérabilité des femmes en question, Paris : Presses de Sciences Po.

«Ce qui compte et ce qui ne compte pas : usages des statistiques et violences faites aux femmes », Cahiers du Genre, HS 2, p. 157-177.

« La "Lolita" et la "sex bomb", figures de socialisation des jeunes filles. L'hypersexualisation en question », Sociologie et sociétés, 43 (1), p. 45-71. 
Lofland L., 1998

Löw M., 2015 [2001]

Mardon A., 2010

Mardon A., 2011

Martin K., 1998

May R., 2014

McMillan T., Day K., Boarnet M. et al., 2006

Pelage A., Brachet S., Brugeilles C., Paillet A., Rollet C., Samuel O., 2016

Pinson G., 2019

Rivière C., 2014

Rivière C., 2016

Rivière C., 2017
The Public Realm. Exploring the City's Quintessential Social Territory, New York (N. Y.) : Aldine De Gruyter.

Sociologie de l'espace, Paris : Éditions de la Maison des sciences de l'homme.

« Pour une analyse de la transition entre enfance et adolescence », Agora débats/jeunesses, 54, p. 13-26.

« La génération Lolita. Stratégies de contrôle et de contournement », Réseaux, 168-169, p. 111-132.

« Becoming a Gendered Body : Practices of Preschools », American Sociological Review, 63, p. 494-511.

Urban Nightlife. Entertaining Race, Class and Culture in Public Space, New Brunswick (N. J.) : Rutgers University Press.

« Johnny Walks to School - Does Jane? Sex Differences in Children's Active Travel to School », Children, Youth and Environments, 16 (1), p. 75-89.

« "Alors c'est quoi, une fille ou un garçon?" Travail de préparation autour du genre pendant la grossesse », Actes de la recherche en sciences sociales, 214 , p. 30-45.

«Penser par cas, penser par comparaison. Études urbaines et pratique des monographies comparées », dans Authier J.-Y., Baggioni V., Cousin B., Fijalkow Y. et Launay L. (dir.), D’une ville à l'autre. La comparaison internationale en sociologie urbaine, Paris : La Découverte, p. 43-66.

Ce que tous les parents disent? Approche compréhensive de l'encadrement parental des pratiques urbaines des enfants en contexte de mixité sociale (Paris-Milan), thèse de doctorat en sociologie, Institut d'Études Politiques de Paris et Università degli studi di Milano-Bicocca.

« "Les temps ont changé." Le déclin de la présence des enfants dans les espaces publics au prisme des souvenirs des parents d'aujourd'hui », Les Annales de la recherche urbaine, 111, p. 6-17.

« La fabrique des dispositions urbaines. Propriétés sociales des parents et socialisation urbaine des enfants », Actes de la recherche en sciences sociales, 216-217, p. 64-79. 
Rivière C., 2018

Robert P., Zauberman R., 2017

Valentine G., 1997

Vermeersch S., Launay L., Charmes E., 2019

Warr M., 1985
« Entre méfiance, prudence et politesse : quand les parents enseignent à leurs enfants comment se conduire dans les espaces publics urbains à Paris et Milan », Enfances familles générations [en ligne], 30.

Du sentiment d'insécurité à l'Etat sécuritaire, Bordeaux : Le bord de l'eau.

«"My Son is a Bit Dizzy." "My Wife is a Bit Soft" : Gender, Children, and Cultures of Parenting ", Gender, place and culture, 4 (1), p. 37-62.

Quitter Paris? Les classes moyennes entre centres et périphéries, Paris : Créaphis.

«Fear or Rape Among Urban Women », Social Problems, 32 (3), p. 238-250. 